\title{
Antioxidative Effects of $\alpha$-Tocopherol and Riboflavin-butyrate in Rats Dosed with Methyl Linoleate Hydroperoxide
}

\author{
Teruo Miyazawa, Chiharu Sato and Takashi Kaneda \\ Department of Food Chemistry, Faculty of Agriculture, \\ Tohoku University, Sendai 980, Japan
}

Received January 6, 1983

The antioxidative effects of dietary $\alpha$-tocopherol (TOC) and riboflavin-tetrabutyrate (RTB) against tissue lipoperoxidation caused by the long-term administration of methyl linoleate hydroperoxide (HPO) to rats were investigated by measuring the spontaneous chemiluminescence (CL) intensities and thiobarbituric acid (TBA) reactants of the liver, lung and heart.

TOC supplementation resulted in the effective decrease of CL intensities and TBA reactants in the three organs, while RTB supplementation led to the definitive decay of both indices of the lung and a significant decrease in the $\mathrm{CL}$ intensity of the heart, as compared with those of rats dosed with HPO and not given any supplemental antioxidant.

Although the activities of glutathione peroxidase and glutathione reductase in the three organs obtained from rats dosed with HPO for 29 days were clearly lower than those of the control rats, both enzyme activities in rats dosed with antioxidants other than HPO were generally maintained at levels almost equal to those of the control rats. Glutathione peroxidase of the liver and heart and glutathione reductase of the liver were shown to be further activated by the simultaneous supplementation of TOC and RTB.

The results indicated that both TOC and RTB effectively act as antioxidants in rats dosed with HPO over a long-term. One of the antioxidative actions of both agents is ascribed to protection of the glutathione peroxidase system in organs undergoing lipoperoxidation caused by the long-term treatment of dietary hydroperoxides.

An oxidized oil intake has resulted in stimulation of lipid peroxidation in several animal tissues. ${ }^{(\sim 3)}$ This action has the typical toxic effect of deteriorating edible oils, which causes several pathological changes such as bleeding and degeneration of the organs and tissues of animals. ${ }^{4}$ The degree of tissue lipoperoxidation induced by the administration of lipoperoxides to rats can be estimated by a chemiluminescent assay of tissue homogenates. ${ }^{1,2}$ The effect of antioxidants on tissue lipoperoxidation caused by dietary hydroperoxides should be interesting because it elucidates the bioantioxidative functions in animals.

In a previous work we have demonstrated that antioxidants, i.e. $d$ - $\alpha$-tocopherol (TOC) and riboflavin-tetrabutyrate (RTB), inhibit tissue lipid peroxidation induced by a shortterm administration of methyl linoleate hydroperoxide (HPO) to rats. ${ }^{1)}$ And the activities of hepatic glutathione peroxidase were found to be elevated by the short-term dosage of HPO irrespective of any antioxidant supplementation. ${ }^{11}$

As an extension of the previous work, we have investigated the effects of TOC and RTB on tissue lipid peroxidation caused by longterm feeding of HPO to rats, for 29 days, and have described the effect of antioxidants on the glutathione peroxidase system.

\section{MATERIALS AND METHODS}

Methy! linoleate hydroperoxide (HPO). Methyl linoleate derived from safflower oil was allowed to autoxidize, and HPO was obtained as described previously. ${ }^{1)}$

Feeding procedures. Male Wistar rats, weighing about $100 \mathrm{~g}$, were purchased from Nihon Rat Co. As shown in Table I, the given amounts of HPO, TOC (Eisai Co.) and RTB (Tokyo Tanabe Co.) diluted with fresh methyl linoleate (ML) were orally administered respectively at $48 \mathrm{hr}$-intervals to rats using a stomach tube for a period of 29 days. The rats of group I (basal diet rats) were 
dosed only with ML and not given the administration components. During the experimentation period, a basal diet (about $20 \mathrm{~g} / \mathrm{rat} /$ day, Type F-2 pellet rations from Funabashi Farm Co.) was given to the rats of each group. The vitamin E content was $50 \mathrm{mg} / \mathrm{kg}$ of diet and the selenium content was $0.42 \mathrm{ppm}$. The vitamin $B_{2}$ content was $10 \mathrm{mg} / \mathrm{kg}$ diet. At the 30th day after the start of administration, five animals of each group were killed by exsanguination via heart puncture. The liver, heart and lung were then excised.

Thiobarbituric acid (TBA) assay. TBA reactants, as a convenient index for tissue lipoperoxide level, were measured by the method of Ohkawa et al..$^{5)}$ as has been described in a previous paper. ${ }^{1)}$ The TBA value was expressed as $\mu \mathrm{mol}$ of malondialdehyde (MDA)/g of wet tissue.

Measurement of tissue chemiluminescence $(C L)$. As has been described previously, ${ }^{1)}$ the $\mathrm{CL}$ intensity was measured using a $5 \mathrm{ml}$ sample of $10 \%(\mathrm{w} / \mathrm{v})$ tissuephysiological saline homogenate in an Chemiluminescence Analyzer OX-7C (Tohoku Electronic Industries Co.). The CL intensity was expressed in terms of the average count/minute for the $200-\mathrm{sec}$ measurement and corrected for background counts.

Estimation of $\alpha$-tocopherol content. The tocopherol content of each tissue was determined by the procedure of Abe et al. ${ }^{\text {b) }}$ using high performance liquid chromatography as has been mentioned previously. ${ }^{1)}$

Assay of glutathione peroxidase system. For the enzyme assay, rats bred in the same condition as described in Table I (groups II $\sim$ V) were used, and control rats, which were fed a basal diet and not dosed with either ML or other administration components, were employed in place of the ML group. The glutathione peroxidase activity of the supernatant fraction, obtained by centrifugation of the homogenate at 105,000 $\times g$, was assayed by the method described previously ${ }^{1)}$ using cumene hydroperoxide as a peroxide donor. The glutathione reductase activity was determined by the method of Racker. ${ }^{7)}$ The specific activities, hereafter called activities, of glutathione peroxidase and glutathione reductase were expressed as $\mu$ mol NADPH oxidized $/ 10 \mathrm{~min} / \mathrm{mg}$ of protein. Protein was assayed by the method of Miller. ${ }^{8)}$

\section{RESULTS}

\section{Antioxidative effects of TOC and RTB in rats dosed with HPO for 29 days}

Figure 1 shows the growth curve. The body weight gains of animals in five groups (groups I V) compared favorably with each other

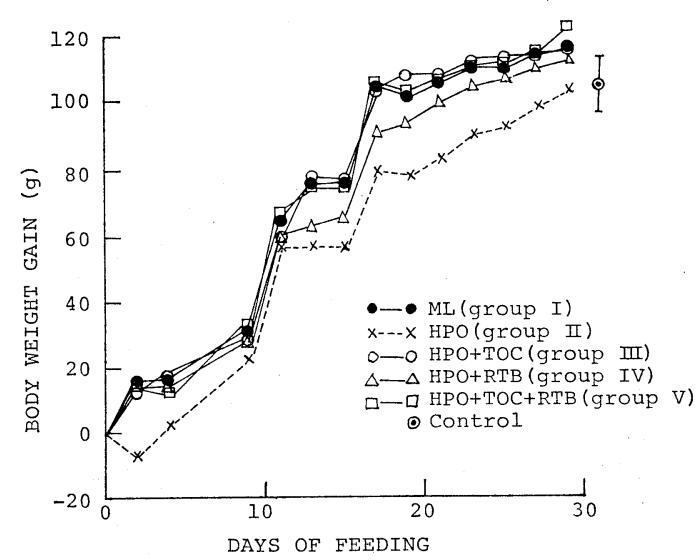

Fig. 1. Body Weight Gains of Rats Dosed with Methyl Linoleate Hydroperoxide and Antioxidants.

Control rats were given neither ML nor supplemental components, and the vertical bar represents the standard deviation. For abbreviations see the legend in Table I. The mean values of body weight gain $(n=5)$ were plotted.

during the 29 day feeding; however the HPO rats (group II) revealed lower body weight gains.

The CL intensities, TBA values and $\alpha$ tocopherol contents of the liver, lung and heart of rats in the five groups are shown in Tables II, III and IV.

Liver As shown in Table II, the CL intensities and TBA values of liver homogenates obtained from the rats of group III and group $\mathrm{V}$ (which had been administered with supplemental TOC other than HPO) were significantly lower than those of the other three groups which had not been given the supplemental

TABLE I. Regimens OF ORALly ADMINISTERED COMPONENTS

\begin{tabular}{llrrrr}
\hline & & \multicolumn{3}{c}{$\begin{array}{c}\text { Administration components } \\
\text { (mg/rat/2 days) }\end{array}$} \\
\cline { 3 - 6 } & Rat group & \\
& & ML & HPO & TOC & RTB \\
\hline I & ML & 200 & - & - & - \\
II & HPO & 200 & 5 & - & - \\
III & HPO+TOC & 200 & 5 & 5 & - \\
IV & HPO+RTB & 200 & 5 & - & 0.1 \\
V & HOP + TOC+RTB & 200 & 5 & 5 & 0.1 \\
\hline
\end{tabular}

a Each group consists of five rats.

$b$ ML, methyl linoleate; HPO, methyl linoleate hydroperoxide; TOC, $d$ - $\alpha$-tocopherol; RTB, riboflavin-2', $3^{\prime}, 4^{\prime}, 5^{\prime}$-tetrabutyrate. 
Table II. Liver Chemiluminescence, TBA Value and Tocopherol Content in Rats Dosed with Methyl Linoleate Hydroperoxide for 29 Days

\begin{tabular}{llccc}
\hline Rat group & $\begin{array}{c}\text { Chemiluminescent } \\
\text { intensity } \\
(\mathrm{cpm})\end{array}$ & $\begin{array}{c}\text { TBA Value } \\
(\mathrm{nmol} \text { MDA/ } \\
\text { g wet wt. })\end{array}$ & $\begin{array}{c}\alpha \text {-Tocopherol } \\
\text { content } \\
(\mu \mathrm{g} / \mathrm{g} \text { wet wt. })\end{array}$ \\
\hline I & ML & $528 \pm 156$ & $704 \pm 77$ & $9.3 \pm 0.7$ \\
II & HPO & $690 \pm 198$ & $693 \pm 22$ & $8.5 \pm 0.7$ \\
III & HPO+TOC & $396 \pm 78^{*}$ & $556 \pm 19^{*}$ & $16.5 \pm 3.2^{*}$ \\
IV & HPO+RTB & $504 \pm 66$ & $638 \pm 66$ & $12.2 \pm 1.9^{*}$ \\
V & HPO+TOC+RTB & $354 \pm 72^{*}$ & $572 \pm 36^{*}$ & $18.9 \pm 1.7^{*}$ \\
\hline
\end{tabular}

Abbreviations are the same as described in Table I.

* Significantly different from group II (HPO rat) at $p<0.05$.

Table III. Lung Chemiluminescence, TBA Value and Tocopherol Content in Rats Dosed with Methyl Linoleate Hydroperoxide for 29 Days

\begin{tabular}{llccc}
\hline Rat group & $\begin{array}{c}\text { Chemiluminescent } \\
\text { intensity } \\
(\mathrm{cpm})\end{array}$ & $\begin{array}{c}\text { TBA Value } \\
(\text { nmol MDA/ } \\
\text { g wet wt. })\end{array}$ & $\begin{array}{c}\alpha \text {-Tocopherol } \\
\text { content } \\
(\mu \mathrm{g} / \mathrm{g} \text { wet wt. })\end{array}$ \\
\hline I & ML & $3348 \pm 678^{*}$ & $437 \pm 33$ & $17.8 \pm 4.0$ \\
II & HPO & $5304 \pm 1154$ & $443 \pm 8$ & $18.1 \pm 0.5$ \\
III & HPO+TOC & $2628 \pm 162^{*}$ & $366 \pm 6^{*}$ & $56.0 \pm 13.1^{*}$ \\
IV & HPO+RTB & $2658 \pm 444^{*}$ & $374 \pm 17^{*}$ & $29.7 \pm 12.3$ \\
V & HPO+TOC+RTB & $2952 \pm 600^{*}$ & $341 \pm 14^{*}$ & $47.8 \pm 25.3^{*}$ \\
\hline
\end{tabular}

Descriptions are the same as in legend of Table II.

Table IV. Heart Chemiluminescence, tBa Value and Tocopherol Content in Rats Dosed with Methyl Linoleate Hydroperoxide for 29 Days

\begin{tabular}{llccc}
\hline Rat group & $\begin{array}{c}\text { Chemiluminescent } \\
\text { intensity } \\
(\mathrm{cpm})\end{array}$ & $\begin{array}{c}\text { TBA Value } \\
(\text { mmol MDA/ } \\
\text { g wet wt. })\end{array}$ & $\begin{array}{c}\alpha \text {-Tocopherol } \\
\text { content } \\
(\mu \mathrm{g} / \mathrm{g} \text { wet wt. })\end{array}$ \\
\hline I & ML & $1668 \pm 348^{*}$ & $754 \pm 94$ & $18.5 \pm 1.2$ \\
II & HPO & $7692 \pm 2292$ & $666 \pm 173$ & $20.6 \pm 1.1$ \\
III & HPO+TOC & $2172 \pm 84^{*}$ & $435 \pm 30^{*}$ & $27.1 \pm 1.8^{*}$ \\
IV & HPO+RTB & $2250 \pm 546^{*}$ & $481 \pm 25$ & $21.3 \pm 2.0$ \\
V & HPO+TOC+RTB & $2070 \pm 378^{*}$ & $470 \pm 39$ & $27.9 \pm 2.4^{*}$ \\
\hline
\end{tabular}

Descriptions are the same as in legend of Table II.

TOC, The $\alpha$-tocopherol contents of the livers of rats in group III and group V were higher than those of HPO rats (group II) and of ML rats (group I). Supplementation of RTB elevated the $\alpha$-tocopherol contents as revealed in the results for group IV. Although the ML rats (group I) were not given HPO, the TBA value was almost at the same level as that of the HPO-dosed rats (group II).

Lung The CL intensities of the lung of ML rats (group I) and of rats supplemented with antioxidants (groups III, IV and V) were noticeably lower than those of HPO rats (group II), as shown in Table III. The TBA reactants of the lung of rats given antioxidants (groups III, IV and V) were less than those of HPO rats (group II) and of ML rats (group I). The $\alpha$-tocopherol contents of the lung were highly elevated in rats supplemented with TOC (groups III and V).

Heart The CL intensities of the heart were markedly increased in HPO rats (group II), and the CL intensities of ML rats (group I) and of rats supplemented with antioxidants (groups III, IV and V) were significantly lower than those of HPO rats (Table IV). The TBA values of the heart of rats in group III, which were given TOC, were clearly lower than those of the other four groups. The $\alpha$-tocopherol contents of the heart were increased in rats 
Table V. Glutathione Peroxidase Activities of Rats Dosed with METHYl LiNOLEATE HYDROPEROXIDE FOR 29 DAYS

\begin{tabular}{|c|c|c|c|c|}
\hline & \multirow{2}{*}{ Rat group } & \multicolumn{3}{|c|}{$\begin{array}{c}\text { Glutathione peroxidase } \\
\text { ( } \mu \mathrm{mol} \mathrm{NADPH} \text { oxidized } / 10 \mathrm{~min} / \mathrm{mg} \text { protein })\end{array}$} \\
\hline & & Liver & Lung & Heart \\
\hline I & Control $^{a}$ & $279.2 \pm 10.5^{*}$ & $242.4 \pm 3.1$ & $248.7 \pm 6.4$ \\
\hline II & $\mathrm{HPO}$ & $192.7 \pm 20.3$ & $229.7 \pm 8.3$ & $229.5 \pm 18.0$ \\
\hline III & $\mathrm{HPO}+\mathrm{TOC}$ & $292.2 \pm 5.2^{*}$ & $231.0 \pm 7.7$ & $235.8 \pm 15.7$ \\
\hline IV & $\mathrm{HPO}+\mathrm{RTB}$ & $274.0 \pm 3.8^{*}$ & $256.3 \pm 3.8^{*}$ & $255.1 \pm 4.7^{*}$ \\
\hline $\mathrm{V}$ & $\mathrm{HPO}+\mathrm{TOC}+\mathrm{RTB}$ & $311.6 \pm 7.0^{*}$ & $261.7 \pm 7.7^{*}$ & $299.5 \pm 12.1^{*}$ \\
\hline
\end{tabular}

Descriptions are the same as in Table II.

a Control rats were fed a standard basal diet (Type F-2 from Funabashi Farms Co.) and given neither of the supplemental components.

Table VI. Glutathione Reductase Activities of Rats Dosed with METHYL LINOLEATE HyDROPEROXIDE FOR 29 DAYS

\begin{tabular}{|c|c|c|c|c|}
\hline & \multirow{2}{*}{ Rat group } & \multicolumn{3}{|c|}{$\begin{array}{c}\text { Glutathione reductase } \\
\text { ( } \mu \mathrm{mol} \text { NADPH oxidized } / 10 \mathrm{~min} / \mathrm{mg} \text { protein) }\end{array}$} \\
\hline & & Liver & Lung & Heart \\
\hline I & Control & $218.5 \pm 7.9^{*}$ & $191.6 \pm 10.9$ & $235.3 \pm 5.9^{*}$ \\
\hline II & HPO & $161.5 \pm 10.0$ & $188.0 \pm 8.6$ & $192.9 \pm 4.8$ \\
\hline III & $\mathrm{HPO}+\mathrm{TOC}$ & $238.8 \pm 5.9^{*}$ & $188.0 \pm 7.1$ & $187.6 \pm 12.8$ \\
\hline IV & $\mathrm{HPO}+\mathrm{RTB}$ & $226.6 \pm 9.0^{*}$ & $223.4 \pm 7.2 *$ & $232.8 \pm 9.1^{*}$ \\
\hline $\mathrm{V}$ & $\mathrm{HPO}+\mathrm{TOC}+\mathrm{RTB}$ & $272.4 \pm 12.9^{*}$ & $244.3 \pm 3.1^{*}$ & $229.9 \pm 11.3^{*}$ \\
\hline
\end{tabular}

Descriptions are the same as in legend of Table V.

given supplemental TOC (groups III and V), similar to those observed for the liver and lung.

\section{Changes of the glutathione peroxidase system} in liver, lung and heart

Changes in the activities of glutathione peroxidase and glutathione reductase in the three organs of rats dosed with HPO and supplemental antioxidants for 29 days are shown in Table V and VI.

The glutathione peroxidase activities of the liver of rats in group II, which had been dosed with HPO and not given any antioxidants, were significantly lower than those of the control rats (group I) as shown in Table V. The hepatic glutathione peroxidase activities of animals supplemented with TOC or RTB (groups III and IV) were held at almost the same levels as those of the control rats. Simultaneous supplementation of TOC and RTB (group V) resulted in further elevation of the hepatic glutathione peroxidase activities. In the lung, the glutathione peroxidase activities of animals supplemented with RTB (groups IV and V) were increased (Table V). In the heart, the glutathione peroxidase activities were elevated in rats given RTB (groups IV and V), and it was also noted that the glutathione peroxidase activities were higher in group $\mathrm{V}$ which had been supplemented with both TOC and RTB (Table V).

The glutathione reductase activities of the liver of rats in group II which had been dosed with HPO and not any antioxidants were lower than those of the control rats (group I) as shown in Table VI. The activities of hepatic glutathione reductase of animals supplemented with either TOC or RTB (groups III and IV) were substantially equal to the level of the control rats. By the simultaneous supplementation of both TOC and RTB (group V), the hepatic glutathione reductase activities were further elevated. In the lung, the reductase activities of animals supplemented with RTB (groups IV and V) were increased, and these activities were higher in group $\mathrm{V}$ which had 
been supplemented with both TOC and RTB (Table VI). In the heart, the glutathione reductase activities of rats supplemented with RTB (groups IV and V) were maintained at the same levels as those of the control rats (Table VI).

\section{DISCUSSION}

The ultraweak CL of systems undergoing lipid peroxidation reflects oxidation reactions involving molecular oxygen, and especially the generation of short-lived free radicals and the excited states derived from the free radical

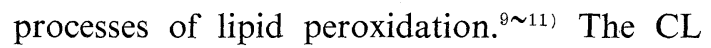
technique permits monitoring of lipoperoxidation reactions occurring in tissue homogenates and has been shown to have an advantage over the detection of lipid peroxides by TBA assay. ${ }^{9,10)}$ During the course of our investigation, ${ }^{1,2)}$ the CL technique has been employed to elucidate the antioxidative properties of TOC and RTB, in addition to the TBA assay.

As revealed before, supplementation of TOC and/or RTB brought about significant decays of CL intensities and TBA reactants of rat organs as compared with those of rats dosed with HPO and not given the supplemental antioxidants (Tables II, III and IV). This shows that both TOC and RTB are demonstrated to be effective antioxidants against tissue lipoperoxidation caused by long-term feeding of dietary hydroperoxides. The relatively higher TBA reactants observed in the organs of $\mathrm{ML}$ rats (Tables II, III and IV) may be explained in a manner similar to when the TBA values rise in the tissues of rats whose dietary linoleic acid triglyceride has been increased. ${ }^{11)}$

By taking these observations and the previous findings ${ }^{1,2)}$ into consideration, the antioxidative nature of both agents among the individual rat tissues may be summarized as follows; TOC is an effective component especially in the liver and kidney against acute toxicity caused by the short-term ( 2 day) feeding of HPO, and TOC is also effective in the liver, heart and lung under a chronic toxicity provided by the long-term ( 29 day) feeding of
HPO. On the other hand, RTB is an effective agent, especially in the liver, lung and heart, at acute intoxication and RTB is also effective in the lung and heart against chronic intoxication with dietary HPO. It is also suggested that the antioxidative functions of dietary TOC are not necessarily the same among all of the organs in animals, and that RTB has the added effect of protecting organs which are not effectively receiving the antioxidative actions of TOC during acute intoxication with HPO. Although the functions and mechanisms for tocopherol as bio-antioxidants have been well studied, ${ }^{13)}$ little is known about the susceptibility of the protective effects on individual organs against HPO-intoxication. The protective effect of $\alpha$-tocopherol is generally regarded as being due to its ability to quench free radicals as well as lipid peroxy radicals in biomembranes. ${ }^{14)}$ It has been stated that $\alpha$ tocopherol, in the process of quenching free radicals, is first oxidized to the tocopheryl radical and subsequently to the tocopheryl quinone, and then the tocopheryl radical is recycled to $\alpha$-tocopherol by the action of various thiols such as reduced glutathione which has been provided by glutathione reductase. ${ }^{15)}$ The higher tocopherol level observed in the liver of rats dosed with HPO and supplemented with RTB (Table II) suggests the protective action of RTB against the consumption of tissue $\alpha$ tocopherol undergoing lipoperoxidation. The protective effect of riboflavins is not as clearly related as that of $\alpha$-tocopherol against the damaging effects of dietary hydroperoxides. It has been noted that the elevation of TBA reactants in blood plasma of riboflavin-deficient diet rats administered with oxidized oil was effectively prevented by the supplementation of riboflavin. ${ }^{16)}$ Because of its hydrophobic structure, RTB is thought to be more effective than the riboflavin free form against tissue deterioration caused by dietary hydroperoxides.

The glutathione peroxidase system, i.e. glutathione peroxidase and linked glutathione reductase, is believed to act on wide varieties of lipid hydroperoxides, reducing them to fatty acid alcohols. ${ }^{18)}$ In addition it is also suggested 
that glutathione peroxidase exerts its protective effect by preventing an attack by free radicals on the lipids, thus preventing lipid peroxidation. ${ }^{19)}$ Under the present conditions, the activities of the glutathione peroxidase system were meaningfully reduced by the longterm administration of HPO when compared with those of the control rats (Table V and VI). This implicates that deactivative events of the glutathione peroxidase system had occurred under the highly propagated lipoperoxidation of tissues upon the administration of HPO to animals. ${ }^{20}$ ) The deactivative effect on the enzyme system was not observed in the organs of rats supplemented with TOC and/or RTB, suggesting one site for the antioxidative functions of both agents. It is reasonably suggested that the production of free radicals by subsequent free radical chain reactions involved in lipid peroxidation leads ultimately to membrane damage and cross-linking of biomolecules, and the net result is a decline in cellular integrity caused by reduced enzyme activities. ${ }^{21)}$ The antioxidative effects of TOC and RTB against intoxication with dietary HPO, which appeared in the present study, may be explained as being due to their protection against membranous damage with lipid free radicals being yielded in the HPO-dosed rats.

The activity of glutathione peroxidase is known to be dependent upon certain dietary factors, notably selenium. Tappel has demonstrated that glutathione peroxidase activity is proportional to the $\log$ of the concentration of selenium in the diet. ${ }^{18)}$ The selenium content, $0.42 \mathrm{ppm}$, of the diet used in the present study is recognized to be sufficient to substantiate the antioxidative effects of TOC and RTB under the present conditions.

\section{REFERENCES}

1) T. Miyazawa, A. Nagaoka and T. Kaneda, Agric. Biol. Chem., 47, 1333 (1983).

2) T. Miyazawa, T. Kaneda, C. Takyu and H. Inaba, J. Nutr. Sci. Vitaminol., 29, 53 (1983).

3) K. Nakatsugawa and T. Kaneda, Yukagaku Zasshi, 30, 74 (1980).

4) M. Yoshioka and T. Kaneda, Yukagaku Zasshi, 23, 321 (1974).

5) H. Ohkawa, N. Ohishi and K. Yagi, Anal. Biochem., 95, 351 (1979).

6) K. Abe, M. Ohmae and G. Katsui, Vitamin, 50, 453 (1976).

7) E. Racker, "Methods in Enzymology," ed. by S. P. Colowick and N. O. Kaplan, Academic Press, New York, 1955, p. 722.

8) G. L. Miller, Anal. Chem., 31, 964 (1959).

9) A. Boveris, E. Cadenas, R. Reiter, M. Filipkowskii, Y. Nakase and B. Chance, Proc. Natl. Acad. Sci. U.S.A., 77, 347 (1980).

10) N. R. DiLuzio and T. E. Stege, Life Sci., 21, 1457 (1977).

11) T. Miyazawa and T. Kaneda, J. Nutr. Sci. Vitaminol., 27, 415 (1981).

12) N. Iritani, E. Fukuda and Y. Kitamura, J. Nutr., 110, 924 (1980).

13) B. E. Leibovitz and B. V. Siegel, J. Gerontol., 35, 45 (1980).

14) H. M. Tinberg and A. A. Barber, J. Nutr., 100, 413 (1970).

15) W. A. Pryor, "Free Radicals in Biology," Vol. 1, ed. by W. A. Pryor, Academic Press, New York, 1976, p. 19.

16) Y. Saito, N. Ohishi and K. Yagi, J. Nutr. Sci. Vitaminol., 27, 17 (1981).

17) K. Tahara, S. Matsuoka and H. Ohama, J. Nutr. Sci. Vitaminol., 20, 81 (1974).

18) A. L. Tappel, Am. J. Clin. Nutr., 27, 960 (1974).

19) P. B. McCay, D. D. Gibson, K. L. Fong and K. R. Hornbrook, Biochim. Biophys. Acta, 431, 459 (1976).

20) A. L. Tappel, Federation Proc., 32, 1870 (1973).

21) O. Igarashi, N. Shoji and K. Kaneko, Eiyo to Shokuryo, 33, 309 (1980). 